UCRL-JC-114015

PREPRINT

High Rate Resistive Plate Chambers: An Inexpensive,

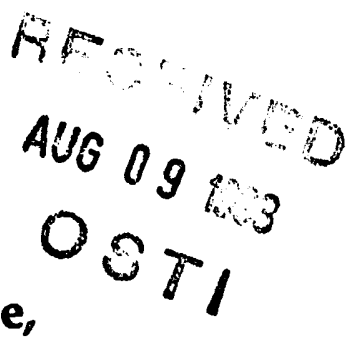
Fast, Large Area Detector of Energetic Charged Particles for Accelerator and Non-Accelerator Applications

C. R. Wuest, E. Ables, R. M. Bionta, O. Clamp, M. Haro,

G. J. Mauger, K. Miller, H. Olsinn, and P. Ramsey

This paper was prepared for submittal to the Proceedings of the Fifth Annual 1993 International Industrial

Symposium on the Supercollider (IISSC)

Plenum Press, San Francisco, California

May 6-8, 1993

May 1993

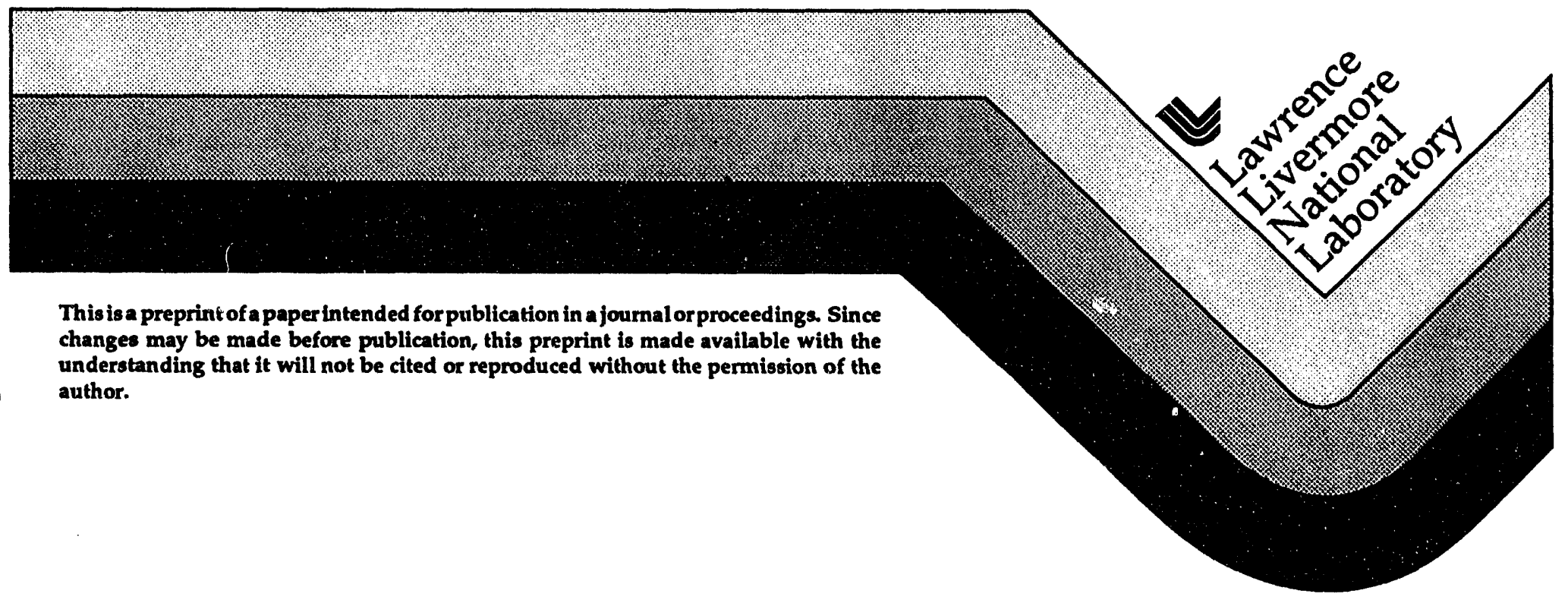


This document was prepared as an account of work sponsored by an agency of the United States Government. Neither the United States Government nor the University of Callfornia nor any of their employees, makes any warranty, express or implied, or assumes any legal liability or responsibility for the accuracy, completenesi, or usefulness of any information, apparatul, product, or process disclosed, or represents that its use would not infringe privately owned rights. Reference henin to any specific commercial product, process, or service by trade name, trademark, manuficturer, or otherwise, cloes not necessarily constitute or imply its endorsement, recommendation, or favoring by the United States Government or the University of California. The views and opinions of author expressed herein do not necessarily state or reflect those of the United States Government or the University of California, and shall not be used for advertising or product endorsement purposes. 


\title{
HIGH RATE RESISTIVE PLATE CHAMBERS: AN INEXPENSIVE, FAST, LARGE AREA DETECTOR OF ENERGETIC CHARGED PARTICLES FOR ACCELERATOR AND NON-ACCELERATOR APPLICATIONS
}

\author{
C. R. Wuest, E. Ables, R. M. Bionta, O. Clamp, M. Haro, G. J. Mauger, \\ K. Miller, H. Olson, P. Ramsey \\ Lawrence Livermore National Laboratory \\ P. O. Box 808 \\ Livermore, CA USA 94551
}

\section{INTRODUCTION}

Resistive Plate Chambers, or RPCs, have been used until recently as large area detectors of cosmic ray muons. They are now finding use as fast large-area trigger and muon detection systems for different high energy physics detectors such the L3 Detector at LEP and future detectors to be built at the Superconducting Super Collider (SSC) and at the Large Hadron Collider (LHC) at CERN. ${ }^{1,2}$ RPC systems at these accelerators must operate with high efficiency, providing nanosecond timing resolution in particle fluences up to a few tens of $\mathrm{kHz} / \mathrm{cm}^{2}$ - with thousands of square meters of active area. RPCs are simple and cheap to construct.

We report here recent work on RPCs using new materials that exhibit a combination of desirable RPC features such as low bulk resistivity, high dielectric strength, low mass, and low cost. These new materials were originally developed for use in electronics assembly areas and other applications, where static electric charge buildup can damage sensitive electrical systems. An accompanying paper presented at this conference, "Behavior of Large Resistive Plate Counters at High Rates," describes complementary work carried out at MIT on "standard" RPCs using resistive materials such as Bakelite. ${ }^{3}$

\section{RESISTIVE PLATE CHAMBER OPERATION}

A sketch of an RPC is shown in Figure 1. It consists of a $2 \mathrm{~mm}$ gas-filled region sandwiched between resistive plates coated with resistive electrodes. An electric potential applied to the electrodes forms a field in the gas of about $4-5 \mathrm{kV} / \mathrm{mm}$. The electrodes typically have surface resistivities of a few $100 \mathrm{k} \Omega / \mathrm{cm}^{2}$. The resistive plates typically have a volume resistivity of $10^{11} \Omega-\mathrm{cm}$ and have been made in the past with plasticized PVC, Bakelite (phenolic), or resistive glass. ${ }^{4-6}$ The passage of a charged particle, such as a muon, causes ionization to occur in the gas, leading to a rapid electrical discharge in the region around the ionization. The resistivity of the electrodes, the plates, and the properties of the gas, act to rapidly quench the discharge, giving very fast pulses with high amplitude. RPC operation is very dependent on the gas mixture. We use RPC gas mixes of argon, isobutane and Freon $13 \mathrm{~B} 1$ in a ratio of about $60 \% / 38 \% / 2 \%$, respectively. The "penalty" one pays for desirable RPC behavior is a corresponding depletion of the electric field near the discharge, leading to inefficient behavior of RPCs at large particle fluxes. The time of the recharge is 


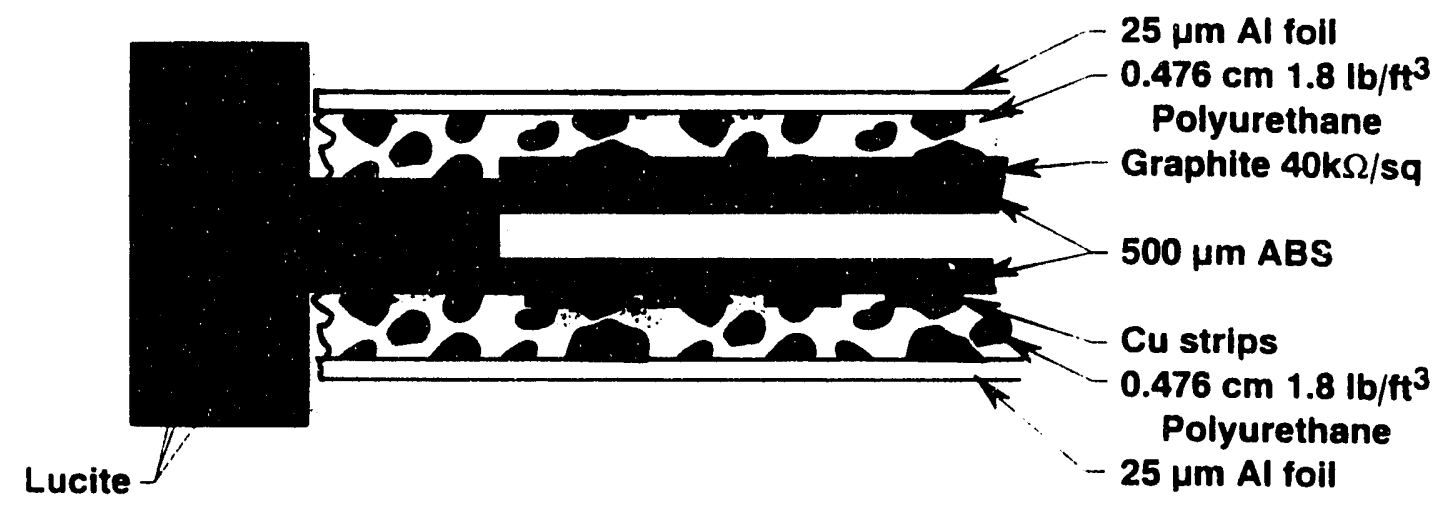

Figure 1. Cross-section of an RPC.

dependent on the resistivity of the RPC plates.

RPC signals are read out using metal pick-up strips, typically $1 \mathrm{~cm}$ wide and a few meters long. Large ( $500 \mathrm{mV}$ into $50 \Omega$ ) output pulses are derived from RPCs that can drive standard electronics such as ADCs, TDCs or discriminators without any preamplification. Timing resolutions have been measured to be less than a few nanoseconds. Proposed RPC-based trigger systems provide fast, coarse position resolution of muon tracks using the signal and known position of the pick-up strip.

\section{EXPERIMENTAL RESULTS}

Reference 3 describes the behavior of "standard" RPCs. The conditions in SSC and LHC environments demand RPCs capable of operating at much higher rates than currently available with standard designs. This is because of the large background of neutrons and charged particles expected at these accelerators. Neutron fluxes of about $10^{4}-10^{5} \mathrm{~Hz} / \mathrm{cm}^{2}$ are expected, e.g., in the GEM detector at standard luminosity. Bakelite RPCs have a measured efficiency for neutrons of about $0.5 \%$, which gives singles rates due to neutrons of about $50-500 \mathrm{~Hz} / \mathrm{cm}^{2}$. However, Bakelite RPCs only have a rate capability, at $97 \%$ efficiency, of about $50-100 \mathrm{~Hz} / \mathrm{cm}^{2}$. Therefore, RPCs used in muon detector systems, must have higher rate capability by a factor of 10-100. Our R\&D program indicates that this rate capability is now understood to be solely a function of the bulk resistivity of the resistive plate material.

To develop a more reliable detector with the high rate capability necessary for proposed detector systems, we have studied alternative materials for RPCs. We have built a number of RPCs out of specially formulated static-dispersive plastic materials that exhibit a number of desirable RPC properties including large amplitude, nanosecond pulses with very low noise and high rate capability. Since the plastic sheets are thin $-500 \mu \mathrm{m}$ or less RPCs using these materials have low mass, with about $0.5 \%$ radiation length per RPC.

Figure 2 shows a SPICE model of an RPC. The resistive plates are modeled as a resistor and capacitor in parallel, with the resistor value determined by the measured bulk resistivity and thickness of the material. Table 1 gives parameters for different RPC materials under study. The gas gap is modeled as a simple capacitor. The model is used to predict the dynamic behavior of the RPC when a spark discharge occurs in the gas gap. A characteristic "recovery" time is predicted, associated with the recharging of the electric field in the plate after the discharge occurs. This recharge time varies widely for different materials. Note that the curves in Fig. 2 show the recharge time of the region near the spark discharge and do not repiesent the signal pulse shape out of the RPC. We define the recovery time as the time between the $10 \%$ and $90 \%$ amplitude points on the curves of Fig. 2 .

The recharge time is directly related to the saturated rate capability of the RPC following a simple model that gives the rate of an RPC at a fixed efficiency given the saturation counting rate. This model usually underestimates the rate for high efficiencies. Let $R s=$ saturation rate, $R e=$ rate at a fixed efficiency $E$ then, $R e=R s(1-E)$. This is only an approximation as it assumes a linear relation between saturation counting rate and the period of 
Table 1. RPC Resistive Materials Properties

\begin{tabular}{lccc}
\hline Material & Thickness $(\mathrm{cm})$ & Bulk Resistivity $(\Omega$-cm $)$ & Arc Resistivity $\left(\Omega\right.$-cm $\left.{ }^{2}\right)$ \\
\hline MIT mirror glass & 0.300 & $5.00 \times 10^{12}$ & $1.50 \times 10^{12}$ \\
LLNL mirror glass & 0.066 & $4.90 \times 10^{12}$ & $3.23 \times 10^{11}$ \\
Kodak glass & 0.123 & $6.42 \times 10^{11}$ & $7.89 \times 10^{10}$ \\
Italian RPC Bakelite & 0.200 & $1.00 \times 10^{11}$ & $2.00 \times 10^{10}$ \\
LLNL Bakelite & 0.161 & $4.50 \times 10^{9}$ & $7.24 \times 10^{8}$ \\
Abstat-M310 plastic & 0.072 & $5.78 \times 10^{9}$ & $4.16 \times 10^{8}$ \\
Abstat-M310 plastic & 0.060 & $5.78 \times 10^{9}$ & $3.47 \times 10^{8}$ \\
MiTech-411 plastic & 0.090 & $2.03 \times 10^{9}$ & $1.83 \times 10^{8}$ \\
MiTech-411 plastic & 0.030 & $2.03 \times 10^{9}$ & $6.19 \times 10^{7}$ \\
Corning 0211 glass & 0.056 & $6.70 \times 10^{7}$ & $3.75 \times 10^{6}$ \\
\hline
\end{tabular}

inefficiency of the counter. Assuming $E=0.95$, a typical value, then for $R s=20 \mathrm{kHz}$ we can assume that the counter will operate with full efficiency at $1 \mathrm{kHz}$. Because of the above approximation this derived rate is actually an underestimate of the real rate.
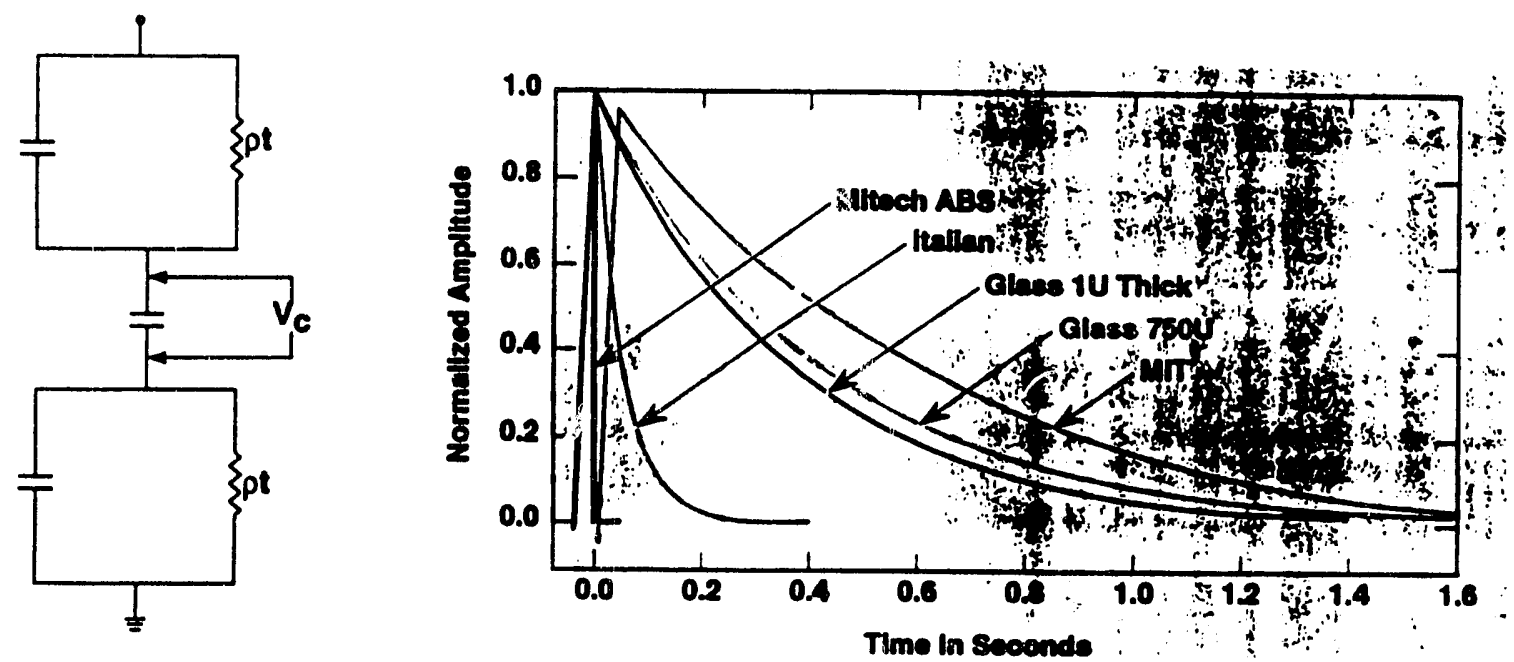

Figure 2. Equivalent circuit and SPICE model predictions of RPC recharge time for various materials. Note that this is not the same as the RPC pulse shape, which is much faster.

Figure 3 gives results for saturated rate measurements for different materials compared to the inverse of the predicted recharge time. The measured rates are proportional to the calculated time response of the material, indicating that the operation of the RPC is understood using this model and the material bulk resistivity. From Fig. 3 we see that two plastics formulated with conducting polymer exhibit very high saturated rate capability compared io glasses and Bakelite, with rates of about $15 \mathrm{kHz} / \mathrm{cm}^{2}$. One plastic is an ABS-based plastic and the other is a PVC-based plastic. We have measured the dielectric strength of the ABS plastic to be superior to the PVC plastic. A number of RPCs constructed from ABS and PVC plastic have been built and tested (the largest being $1.2 \mathrm{~m} \times 2.4 \mathrm{~m}$ ).

Results from measurements of different RPCs indicate that plastic RPCs operate identically to Bakelite RPCs with regards to pulse width, pulse height, and rise-time jitter. In addition, noise measurements with plastic RPCs give a noise rate of about $0.5 \mathrm{~Hz} / \mathrm{cm}^{2}$ for a 


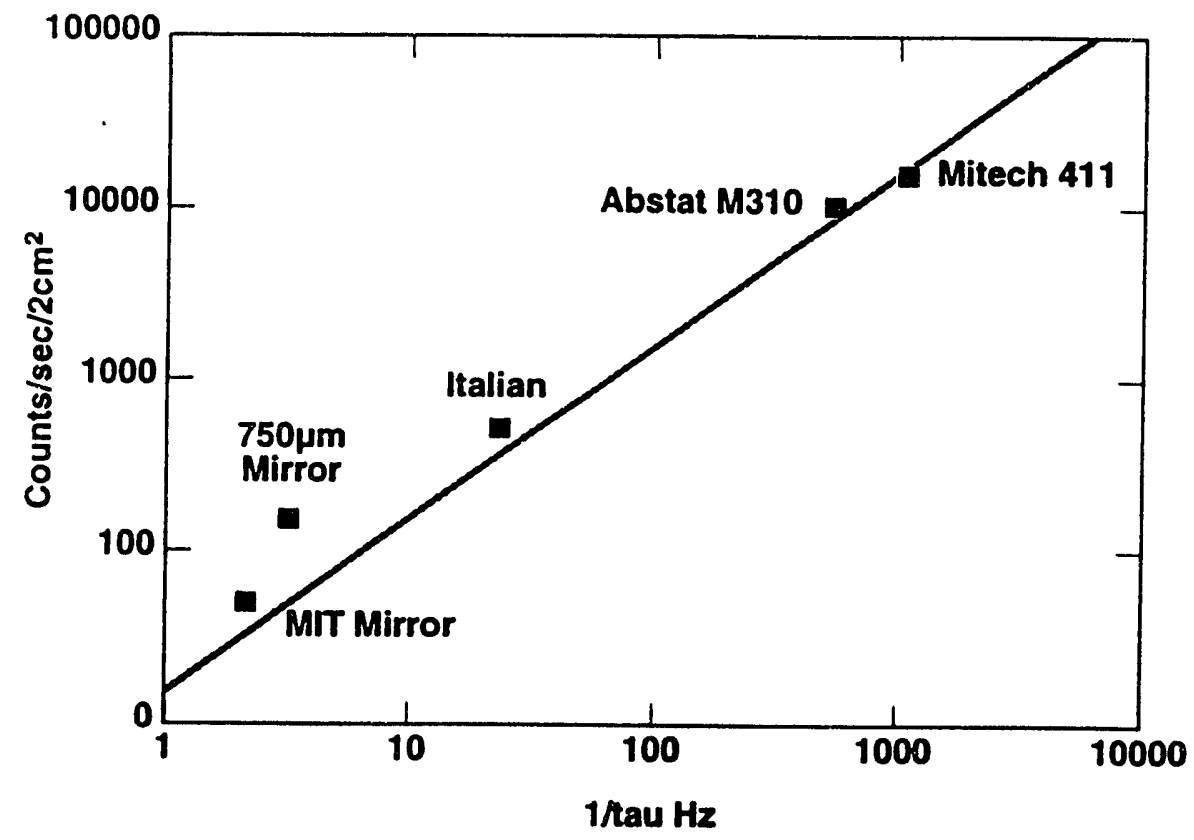

Figure 3. Comparison of SPICE calculated RPC rate capability with measured rates for a number of different RPCs. The RPCs plotted are, in order of increasing rate, MIT glass, LLNL glass, Italian Bakelite, Abstat-M310, and MiTech-411. The linear fit to the points is also indicated.

$15 \mathrm{mV}$ discriminator threshold. This is achieved for plastic sheet without any special surface preparation, compared to Bakelite chambers, which require a special coating of linseed oil on the inner surfaces in order to operate properly.

The ABS RPC does exhibit some variability in operation associated with initial turnon and subsequent voltage changes. This is manifested as a decrease in noise rate over a characteristic settling time of the order of hundreds of minutes. Once the RPC is stabilized the noise rate is the value quoted above. Another characteristic of the ABS plastic is a variability in saturated count rate associated with turn-on and subsequent voltage changes. This appears to be associated with changing bulk resistivity of this particular plastic and is possibly due to polarization, or temperature/humidity effects. We have seen that the saturated rate capability of an ABS RPC is reduced from about $10 \mathrm{kHz} / \mathrm{cm}^{2}$ to about $1 \mathrm{kHz} / \mathrm{cm}^{2}$ over a period of a few hours. At this point the RPC is stable, as shown by measurements with constant yoltage over a 6-day period.

There are a number of other semiconducting plastics (including epoxy paints) that are being studied and it is expected that the variability discussed above will be solved by the choice of the proper material. Plastics with even lower bulk resistivities (about $10^{8} \mathrm{ohm}$ $\mathrm{cm}$ ) have recently been identified. Based on our understanding of RPC operation, these lower resistivity plastics will provide even better RPC operation with correspondingly higher saturated rate capability - by as much as a factor of ten over ABS plastic.

\section{ACKNOWLEDGEMENTS}

This work was performed under the auspices of the US Department of Energy by the Lawrence Livermore National Laboratory under Contract W-7405-ENG-48.

\section{REFERENCES}

1. GEM Collaboration "An Expression of Interest to Construct a Major SSC Detector," GEM-TN-91-1.

2. A. Bohrer, et al., "Status Report of the RD5 Experiment," CERN/DRDC/91-53, January 13, 1992.

3. M. Widgoff, et al., "Behavior of Large Resistive Plate Counters at High Rates," presented at the Fifth International Industrial Symposium on the Super Collider (IISSC), San Francisco, May 7 (1993).

4. G. Battistoni, et al., "Plastic Spark Counters with PVC Electrodes," Nucl. Inst. Meth. A270, p. 190, 1988.

5. R. Cardarelli, et al., "Progress in Resistive Plate Counters," Nucl. Inst. Meth. A263, p. 20, 1988.

6. M. Anelli, et al., "Glass Electrode Spark Counters," Nucl. Inst. Meth. A300, p. 572, 1991. 


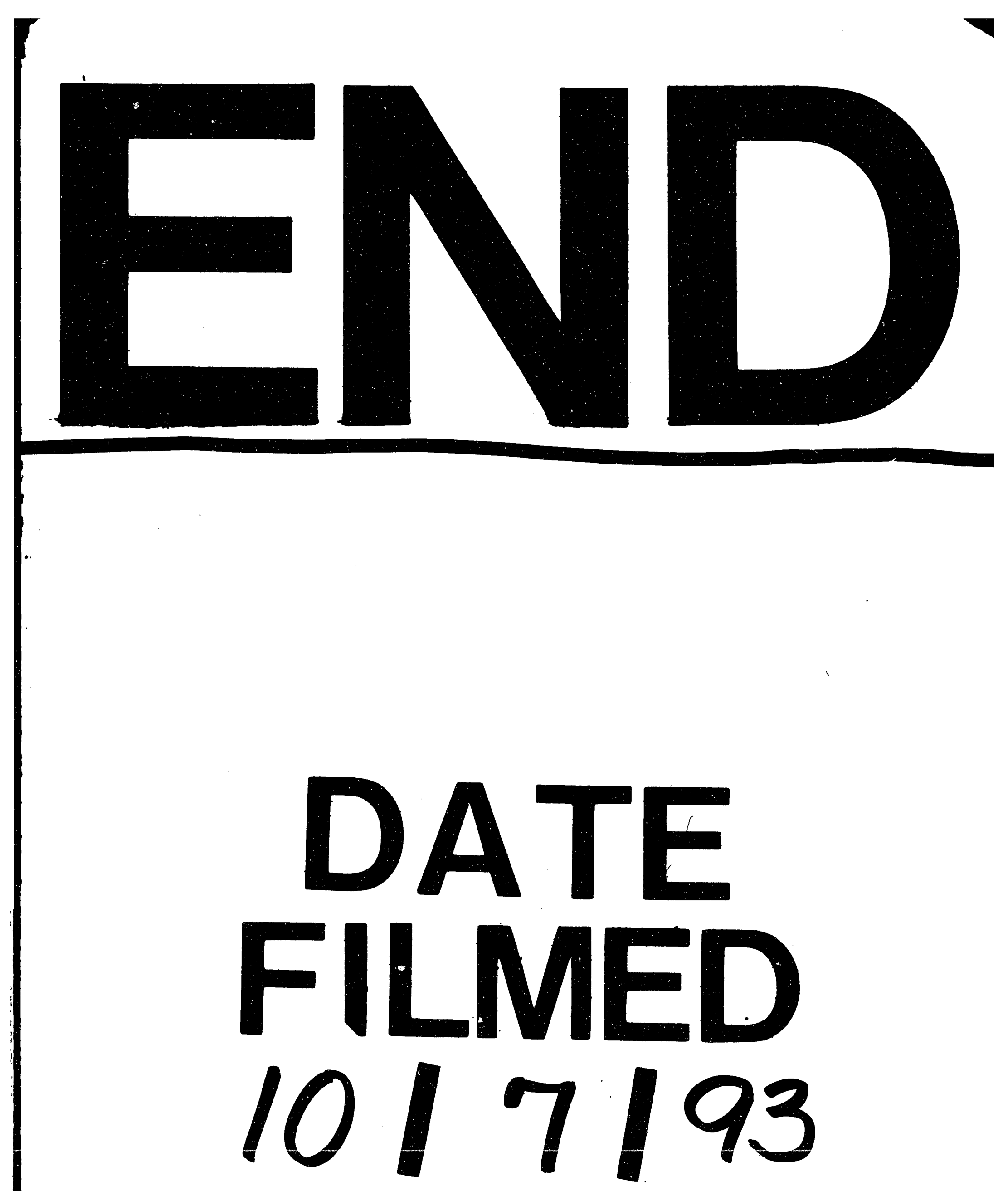




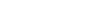

